Check for updates

The BMJ

Cite this as: BMJ 2021;375:n3042 http://dx.doi.org/10.1136/bmi.n3042 Published: 08 December 2021

\title{
Covid-19: Coroners call for changes after highlighting avoidable deaths during pandemic
}

\section{Clare Dyer}

Coroners in England are demanding changes in a series of reports highlighting how the struggling healthcare system's responses to the pressures of the covid-19 pandemic contributed to patients' deaths.

Coroners are obliged to write a report recommending action in any cases where they believe that this is necessary to prevent future deaths. Reports now emerging suggest that factors in deaths during the pandemic include the move by GPs to telephone consultations, the requirement for vulnerable patients to attend hospital appointments alone, and the lack of safeguards for patients in care homes.

The replacement of in-person appointments by telephone consultations reduced GPs' ability to pinpoint patients' needs, the coroners said, and the absence of family members from consultations with vulnerable patients meant that clinicians were often unable to get a full picture of their needs.

The Yorkshire and Humber region saw an increased incidence of children with severe nutritional anaemia in 2020, resulting in two deaths. Maya Zab, who died aged 11 months, was one of the two.

Language barriers had caused missed opportunities for primary carers to see Maya, but this was compounded by the pandemic, said Ian Pears, coroner. The "stay at home" message resulted in fewer one-to-one consultations and meant that healthcare professionals were unable to spot signs of her condition, while the limitation of social contact meant that other professionals and friends were unable to report concerns.

Maurice Leech, who was frail and elderly, had an accidental fall at his nursing home, but there was a delay in recognising that he had a fractured femur. He was reviewed by telephone by his GP but not examined because of covid restrictions. Alison Mutch, senior coroner for Greater Manchester South, said the evidence indicated that a physical examination would probably have resulted in his being referred back to hospital sooner.

"Very vulnerable and a poor historian," she said, Leech was sent alone to hospital when, if support had been available, a more accurate picture of his needs would have helped staff to treat him and potentially identify that he should not be discharged back to the care home.

\section{Care homes}

A policy of reserving vaccines for hospital staff rather than for patients may have contributed to the death of Clive Rivers, who was being treated for a skin condition with immunomodulatory therapy. The consultant dermatologist treating him wanted him to be vaccinated, but it was not policy at that time to vaccinate patients, and Rivers contracted covid-19 in hospital while awaiting discharge. He deteriorated and died at home.

The patients who caught covid-19 in care homes included Anthony Slack, who had asbestos related pulmonary fibrosis and other health issues. The care home managers were unsure whether the virus was brought in by staff or by residents admitted from the community without testing. There was no risk assessment when admitting new residents, and staff were not clear about requirements for personal protective equipment (PPE) as a result of regular changes to guidance, said Mutch, who was also the coroner in Slack's case and several others.

Another issue highlighted was the shortage of staff to monitor frail and vulnerable patients who had to be isolated in their rooms at care homes when suspected of having covid-19. Ruth Jones, who was prone to falls, fell while unobserved in self-isolation and had to be admitted to hospital. Her covid-19 test was negative, but she deteriorated and died of bronchopneumonia exacerbated by a fracture.

"It was unclear how homes were being advised to safely manage residents at risk of falls where isolation was required," said Mutch. "The home were unaware of any guidance that they should follow to manage the risk." 